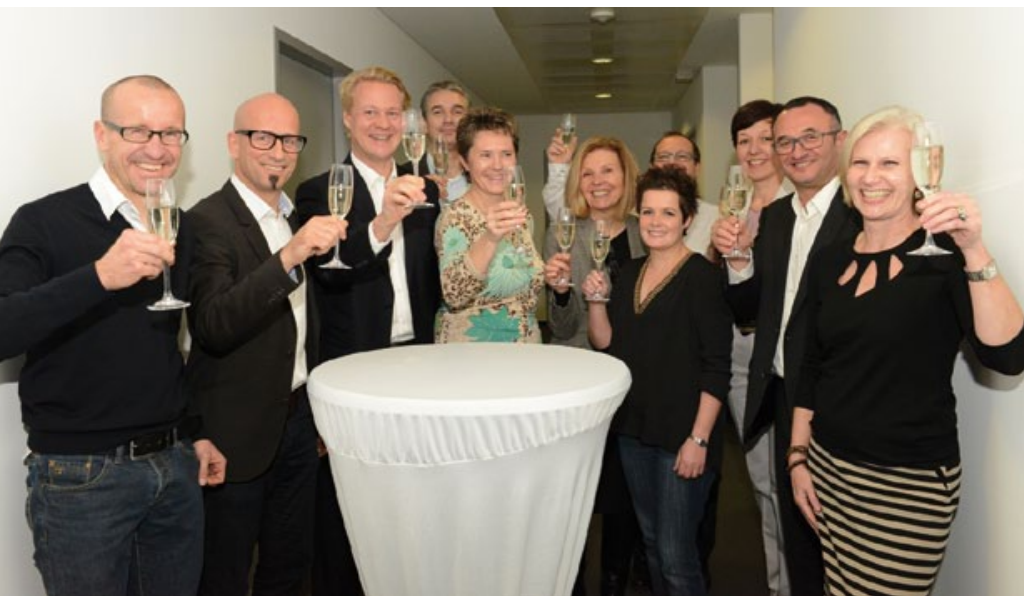

Feierliche Einweihung des neuen Münchner Unternehmenssitzes der BIOMET 3i Deutschland GmbH. Von links: Dr. O. Zuhr, Prof. Dr. M. Hürzeler, Dr. C. Blecker mit Gästen und K. Strauß (vorne rechts).

BIOMET 3i hat mit der deutschen Tochtergesellschaft BIOMET 3i Deutschland $\mathrm{GmbH}$ einen neuen Standort in München bezogen. Am 17. Dezember 2013 wurde der neue Unternehmenssitz im Stadtteil Schwabing feierlich eingeweiht. Zur Eröffnungszeremonie begrüßten die Gast- geber Dr. Carsten Blecker, Vice-President Europe, Middle East \& Africa, und Krista Strauß, General Manager Central Area, Kollegen aus dem In- und Ausland sowie Kunden aus der Region. Zu den Gästen zählten unter anderem die Zahnärzte Prof. Dr. Markus Hürzeler und sein Pra- xispartner Dr. Otto Zuhr, die den Umzug des Unternehmens nach München explizit begrüßten.

Krista Strauß ist die neue Hausherrin am Münchner Standort. Der Umzug des Unternehmens nach München ermöglicht aus ihrer Sicht viele Vorteile für BIOMET 3i und seine Kunden: „Die Standortverlagerung war eine sinnvolle strategische Entscheidung, die unseren Wachstumskurs in Deutschland unterstützt. Die Wahl fiel auf München, weil diese Stadt ein exzellenter Businessstandort mit internationaler Anbindung ist. Außerdem nutzen wir hier die räumliche Nähe zu unseren Kunden in Süddeutschland sowie zu den Nachbarländern Österreich und Schweiz, die zusammen mit Deutschland die Central Area von BIOMET 3i bilden.“

Nach einer Pressemitteilung der

BIOMET 3i Deutschland GmbH, München

\title{
Verleihung des 1. SCAD VITA Award
}

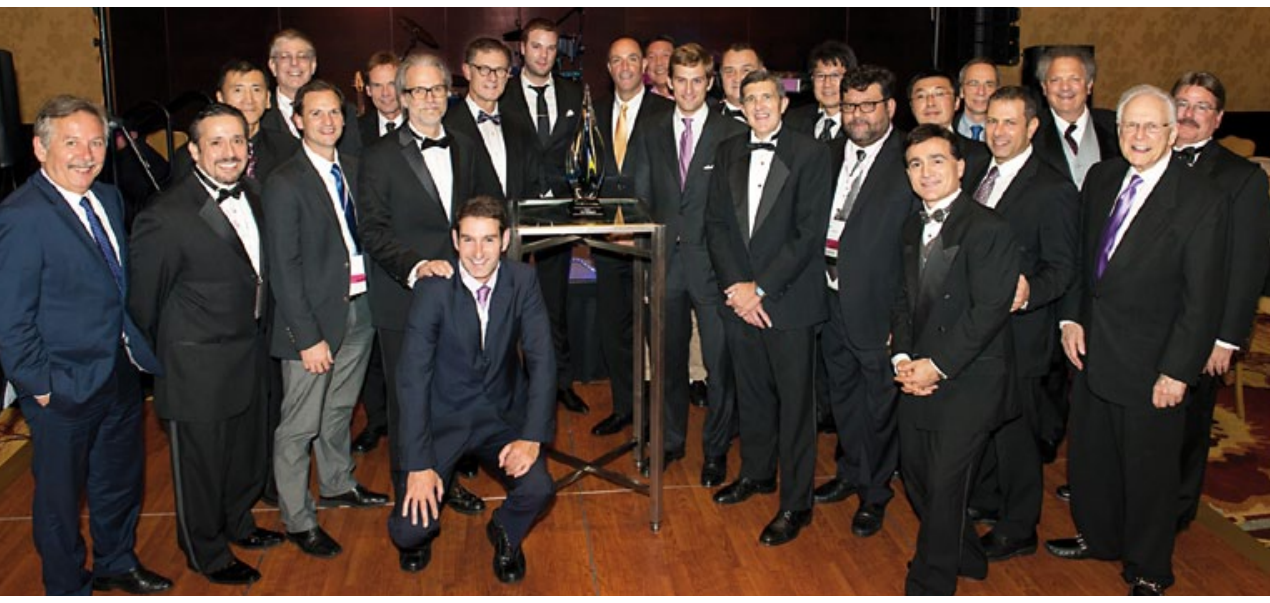

Vom 3.-5. Oktober 2013 fand in der USMetropole Denver der 5. Jahreskongress der Society for Color and Appearance in Dentistry (SCAD) statt. Im Fokus stand ein umfassender Informationsaustausch $\mathrm{zu}$ zahnfarbrelevanten Ästhetikfragen. Neben dem Vortragsprogramm erwartete die Teilnehmer eine Posterausstellung mit insgesamt 24 Beiträgen von Universitäten rund um den Globus, darunter auch die Gewinner der 1. SCAD VITA Award-Aus- schreibung. Diese Auszeichnung wurde in Anerkennung der langjährigen intensiven Unterstützung durch die VITA Zahnfabrik von der SCAD ins Leben gerufen.

Gewinner des 2013 SCAD VITA Award in der Kategorie Young Faculty ist R. Ghinea (Universität Granada, Spanien/Universität Texas, USA) mit ihrer Arbeit über die Aufteilung des dentalen Chromaspektrums in dreidimensionale Unterbereiche gemäß den Farben des Systems VITAPAN classical A1-D4. In der Kategorie Undergraduate Student überzeugte J. Oei (Universität Texas, USA) die Jury mit der Entwicklung eines farbstabilen dualhärtenden UDMA-basierten Restaurationsmaterials mit geringem Chroma. In der Kategorie Graduate Student wurde Dr. A. Kim (Universität Illinois, USA) ausgezeichnet. Sie untersuchte, welche Auswirkungen das Abutmentmaterial auf die Farbe der periimplantären Gingiva und das gesamtästhetische Erscheinungsbild hat. Bewerbungen für den SCAD VITA Award im folgenden Jahr können bis zum 15. August 2014 eingereicht werden. Weitere Informationen hierzu werden unter www.scadent.org zur Verfügung gestellt. Die Gewinner erwartet ein Stipendium in Höhe von 1500,-US-\$. Die Siegerehrung und die Präsentation der ausgezeichneten Projekte erfolgt im Rahmen der 6. Jahreskonferenz der SCAD vom 2.-4. Oktober 2014 im Hotel Sofitel in Chicago zu der die Gewinner eingeladen sind.

Nach einer Pressemitteilung der

VITA Zahnfabrik H. Rauter GmbH \& Co. KG,

Bad Säckingen 


\section{Umweltfreundlich}

\section{FSC-zertifiziertes}

\section{Packmaterial}

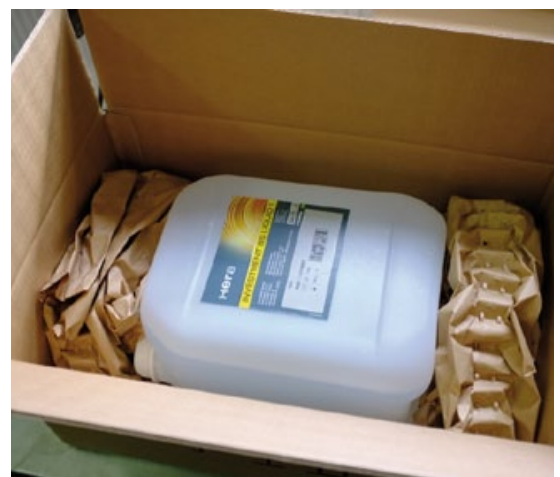

Mit umweltfreundlichem Verpackungsmaterial setzt sich Heraeus Kulzer jetzt für nachhaltige Forstwirtschaft ein: Der Dentalhersteller verwendet ab sofort beim Versand seiner Gipse und Einbettmassen an die Kunden umweltfreundliches, FSC-zertifiziertes Papier als Verpackungsmaterial. „Ich freue mich über diese Neuerung, denn damit leisten wir einen Beitrag zu nachhaltigerem Wirtschaften - ein kleiner, wenn auch wichtiger Schritt“, so Reiner Prystawik, Produktmanager Dental Labor Verbrauchsmaterialien bei Heraeus Kulzer. Die Umstellung kommt an: „Für uns macht es keinen Unterschied, womit die Produkte sicher verpackt sind - ob mit Styroporchips oder Papier. Deshalb finde ich es gut, dass das Unternehmen ein Statement setzt und auf umweltfreundliches Papier umgestiegen ist“, unterstützt Lothar Katnawatos, Zahntechnikermeister und Wiesbadener Kunde des Unternehmens, den eingeschlagenen Kurs.

In Deutschland gründete sich FSC als gemeinnütziger Verein im Jahr 1997. Hinter FSC verbirgt sich ein internationales Zertifizierungssystem mit 10 weltweit gültigen Prinzipien, die in einem internationalen Standard festgelegt sind. Sie garantieren, dass Papiere aus verantwortungsvollen Quellen stammen, also die Ressource Holz aus nachhaltiger Forstwirtschaft gewonnen wird. Neben dem ökologischen Aspekt stellt der FSC-Standard auch die Einhaltung sozialer Rahmenbedingungen wie die Arbeitssicherheit sicher.

Nach einer Pressemitteilung der Heraeus Kulzer GmbH, Hanau Internet: www.heraeus-kulzer.com

\section{Verstärkung im Marketing und Außendienst}

Die Düsseldorfer ARGEN Dental GmbH hat sich zum Jahresanfang 2014 personell verstärkt. Neu geschaffen wurde die Position des Leiters Marketing; zusätzlich nehmen 2 neue Außendienstmitarbeiter für die Region Süddeutschland ihren Dienst auf. Mit der Leitung des Marketings wurde kein Unbekannter in der Dentalbranche betraut: Uwe Heermann war viele Jahre bundesweit als Berater für Dentalindustrieunternehmen und Dentallabore tätig. Mit seiner profunden Erfahrung widmet er sich bei ARGEN Dental seit dem 1. Januar 2014 dem Marketing sowie innovativen Technologien und der Verarbeitung neuer Materialien.

Das Außendienstteam Süddeutschland verstärken Roland Kapitza und Wieland Sterns. Roland Kapitza hat die Zuständigkeit für die Regionen Niederbayern und

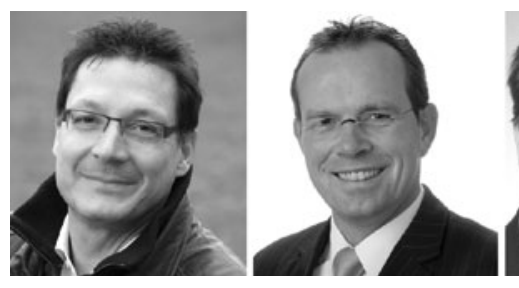

V.I.n. r. W. Sterns, U. Heermann, R. Kapitza

Oberpfalz übernommen, Wieland Sterns für Oberbayern. „Wir sind stolz, erneut 2 technisch erfahrene Außendienstmitarbeiter in unserem Team begrüßen zu dürfen, und freuen uns auf die langfristige Zusammenarbeit“, sagt Hans Hanssen, Geschäftsführer der ARGEN Dental $\mathrm{GmbH}$.

Nach einer Pressemitteilung der ARGEN Dental GmbH, Düsseldorf Internet: www.argen.de

\section{Zahnerhaltung \\ Das Angebot ist rund}

Bei der Fußball-WM gehen nur die Besten an den Start. Deshalb schickt auch Komet seine Favoriten ins Rennen: den K1SM, den beliebten Rosenbohrer aus der CeraLine. $30 \mathrm{~K} 1 \mathrm{SM}$ gibt es jetzt zum Vorzugspreis und einen Komet-Lederfußball gibt es gratis dazu, damit das WMFieber noch mehr Spaß macht. Warum wählte man beim Unternehmen gerade den Spezialisten für minimales Exkavieren? Weil der K1SM bei Zahnärzten Begeisterung auslöst - und das kommt dem Fußballsport doch sehr nahe. Der weiße Keramikbohrer ermöglicht ein kontrolliertes, taktiles Exkavieren. Der Anwender kann spüren, wenn er das kariöse weiche Dentin verlässt. Damit ist der K1SM die direkte Verbindung vom Arzt zur Kavität.

Auch für den vibrationsarmen Hartmetall-Rosenbohrer H1SE / M (mit oder ohne schlanken Hals) hat Komet ein interessantes Angebot zur Fußball-WM. Nie war es günstiger, den Behandlungskomfort durch die einzigartige Hybridverzahnung

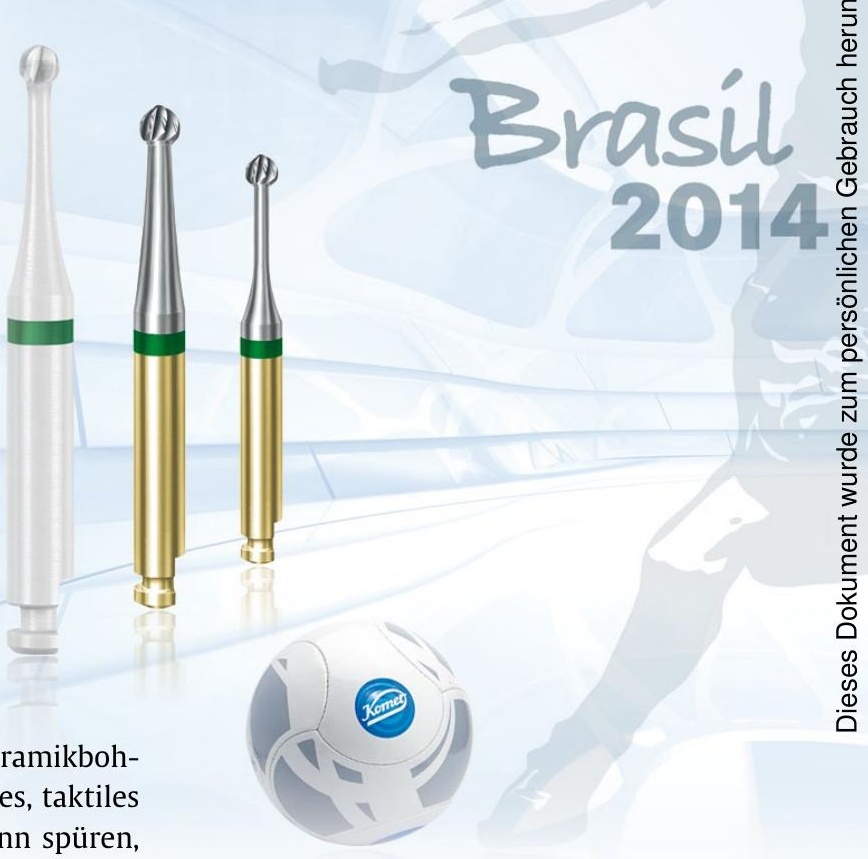

mit 50 Stück zum Vorzugspreis zu erleben. Auch hier gibt es den Komet-Lederfußball gratis dazu. Denn was eint den H1SE / M mit der WM: Schnelligkeit!

Nach einer Pressemitteilung der

Komet Dental Gebr. Brasseler GmbH \& Co KG, Lemgo

Internet: www.kometdental.de 